\title{
Rules of Retinotectal Mapmaking
}

Stephen S. Easter, Jr

\section{Summary}

The selective connections that nerve cells make to each other and to effector and receptor cells outside the nervous system are essential to organized behavior. The retinotectal projection has been used frequently in the experimental investigation of how such patterned sets of connections are formed. This article traces the evolution of some of the dominant experimental paradigms and concepts that have resulted in the currently accepted rules underlying the retinotectal map.

\section{Introduction}

A given nerve cell must have specific connections if it is to share its purpose as an element in a complex network. As an example, consider the motor neurons in our spinal cord. They receive input from a variety of sources, and send their output - trains of action potentials - to the synapses on a few muscle fibers in one muscle. The motor neuron is one half of the simplest of all reflex arcs, the monosynaptic 'stretch reflex', the other half of which is a specialized 'stretch receptor' in the muscle. When the muscle is stretched, the stretch receptor is excited and sends a train of action potentials that excite the motor neuron, which in turn sends a train of action potentials back to the muscle to excite the muscle cells and make them contract. Thus stretch of the muscle leads to contraction of the same muscle. This apparently simple reflex depends essentially on the correctness of the synaptic connections from stretch receptor to motor neuron and from motor neuron to muscle. Should the stretch receptor connect to a motor neuron unconnected to the stretched muscle, then the reflex would be very different, and very likely maladaptive.

In more complex systems, such as peripheral ganglia and various regions in the brain, precise connections have been amply demonstrated. The largest body of work has come from the retinotectal projection of fish, amphibians and birds, and has recently been reviewed by Beazley. ${ }^{1}$

\section{Retinotectal Projection}

The retina, a thin laminar structure on the inside of the eye, responds to light and begins the analysis of the visual stimulus. It is a complex network of at least six different types of neurons, and many more subtypes. The last one to get the message is the ganglion cell, whose axons (tens to hundreds of thousands depending on the animal) form the optic nerve. They enter the brain and terminate in a variety of sites, the major one of which is the optic tectum, a hemispheric laminated structure like the retina. The terminal arbor, the spray of cellular processes at which an optic axon's message is transmitted synaptically to the tectal cells, is deployed at a particular position in the tectum. The ganglion cell's functional type (whether it responds to the onset of light or to a moving stimulus, etc.) probably dictates the depth at which it terminates, but the retinal address of the cell dictates the tectal address of the terminal arbor. The retinotectal projection is usually studied as the solution to a two-dimensional mapping problem, from the retinal cell body to the tectal terminal arbor. It is conveniently simplified if one thinks of the two hemispheric surfaces as twodimensional flattened disks, with positions specified by the polar coordinates, $r$ and $\theta$. The empirical rules of deployment are simple. First, terminal arbors from neighboring retinal ganglion cells lie near one another. Secondly, there is a polarity to the projection, e.g. temporal retinal cells have terminal arbors in rostral tectum, ventral retinal cells have their arbors in dorsal tectum, etc. Thus the projection is topographic and continuous, in the topological sense. Note that these two empirical rules relate the addresses of the two ends of the retinal ganglion cells, but do not speak to the issue of how the map was achieved.

\section{The Chemoaffinity Hypothesis}

These empirical rules have been confirmed in excruciating detail by a variety of investigators in a variety of vertebrates, particularly fish and amphibians. (Similar topographic projections exist in many invertebrates, too, but they have received much less attention.) Roger Sperry, of Cal Tech, can fairly be said to have initiated the interest in the retinotectal projection, and while his Nobel Prize (shared with David Hubel and Torsten Wiesel) was awarded nominally for his work on mammalian cerebral cortex, his contribution to developmental neurobiology was at least as significant, in the minds of many. He realized that the orderly projection between these two hemispheres was a specific example of a generally important feature of the nervous system, and he sought to understand it. His initial experiments were behavioral, the later ones anatomical. They were unfailingly well conceived and cleverly executed, and they culminated in his celebrated hypothesis of chemoaffinity (or chemospecificity). ${ }^{2}$

According to this idea, particular neurons preferentially form connections with others on the basis of chemical markers, presumed to be in the membranes of the two cells. Thus a particular retinal ganglion cell from temporal retina has an identifying marker molecule (or molecules) in the leading edge of the growth cone, the tip of the process that it sends toward the tectum. Once in the tectum, the growth cone may contact a number of tectal cells, but make functional contact with only one or a few of them, chosen because they possess marker molecules that form stable bonds with those on the growth cone. The details of these marker molecules were never spelled out; indeed they were hypothetical constructs, and much of the attraction of the hypothesis was that it suggested what the biochemists ought to search for, if neural development was to be understood in molecular terms.

The chemoaffinity hypothesis reigned supreme for many years, and provided an indisputably better explanation for a lot of developmental phenomena than its predecessors, many of which were more psychological than biological in flavor. The chemoaffinity hypothesis may have been the first successful attempt to force neuroscientists to consider their field as a subdivision of 
REVIEW ARTICLES

(cell) biology. Indeed, the neuroanatomists had accepted the cell as the basic building block of the nervous system very late. Long after the cell doctrine had been accepted for other tissues, neuroanatomists continued to debate the merits of 'neuronism' (the neuron doctrine) vs. 'reticularism' (the syncytial alternative).

\section{Variant Hypotheses}

More recent experiments have led to the conclusion that chemoaffinity is less important to neural development than previously thought. ${ }^{3}$ Most workers in the field continue to accept that something like chemoaffinity must exist, but as one of several important influences rather than the pre-eminent one. Learned and heated discussions of the theory turned on different interpretations of its many formulations. There seems no point in reopening these discussions, as the recent history suggests that a precise meaning of the theory would be difficult to agree on. Suffice it to say that the idea of selective intercellular affinities is alive and well, and believed to be one of several determinants of ordered connections.

This belief is still based more on faith than on observations. The two most popular conceptions of how the chemical markers might work are these: either each cell (or small group of cells) is (are) marked by an identifying molecular species different from the one identifying cells at other locations; or the amount (rather than the kind) of some marker molecule varies with position. The first of these alternatives, analogous to an immunological mechanism, has received no experimental support. The second alternative has. The TOP (for toponymic) antigen of Trisler et al.$^{4}$ is on all cells in the embryonic chick retina, and antibody binding to it increases monotonically over a 35-fold range along a line oriented roughly dorsoventrally. This is just the behavior that one hoped to see in a chemical marker of retinal position. But three observations suggest caution. First, a comparable antigen for the temporonasal direction has not been found; such an antigen would be necessary for a two-dimensional addressing system. Secondly, the TOP antigen is not expressed in the tectum, so a homophilic binding mechanism cannot be involved. Thirdly, experiments with the explanted chick retinotectal system have failed to show any difference of pathfinding between axons from different locations along the dorsoventral axis, although similar experi- ments have shown differences between axons from different nasotemporal positions. $^{5}$ Thus axonal pathfinding varies with retinal position where there are no known positionally variable molecules, but along the axis in which a molecule has been identified the behavior does not vary. The final word is not yet in on TOP, but the story is apparently not as simple as originally guessed.

Another attempt to put neuronal connections on a more chemical basis has come from Edelman and his collaborators. Their discovery and characterization of the neural cell adhesion molecule (N-CAM) enabled them to offer very concrete hypotheses about its function. In one of these, ${ }^{6}$ it was proposed that a variety of markers were not necessary, that a single marker, $\mathrm{N}$-CAM, would suffice if its abundance and binding strength were spatiotemporally modulated. The role of CAMs in neural development remains to be clarified.

Because the 'chemo-' has proved so difficult to establish in chemoaffinity, most progress in understanding the formation of the retinotectal map has come from observations at the cellular, rather than the molecular, level. In the remaining space, I will summarize some of this work, with an emphasis on those aspects that have interested me the most, and on which my own work has centered.

\section{New Observations}

Two phases characterize the process of retinotectal mapmaking. The initial axonal outgrowth results in the formation of the first connections, and evidence from the amphibian Xenopus laevis indicates that these connections are ordered from the beginning in a fashion that matches the adult arrangement. Subsequently, over a period of years, more optic axons grow in and enlarge the projection. The first phase involves tens to perhaps hundreds of axons. The second phase involves hundreds of thousands. The existence of the second phase, its extended duration, and some complexities associated with it, have been appreciated only fairly recently.

The first axons grow out from the presumptive Xenopus retina at a time when the nervous system is quite primitive. Most of the cells in the retinal field are still dividing, but a few, in what will become dorsal retina, stop dividing and begin to differentiate. Their axonal growth cones advance medially along the optic stalk, cross the midline, and enter the presumptive tectum, a patch only about $100 \mu \mathrm{m}$ wide. Evidence that the initial projection is ordered has come from three sorts of experiments. In the first, "compound eyes' were made; these are surgical mosaics in which half of the host's eye anlage is removed, and the remaining half has added to it a second half taken from an inappropriate location of a donor eye. Thus, a 'double temporal' eye (TT) can be made by removing the nasal half of the host's eye anlage and substituting for it the temporal half from a donor. Such an eye has a restricted set of origins, and if temporal axons tended preferentially to innervate the rostral tectum from the outset, then the early projection of a TT eye would be expected to be restricted to rostral tectum even though half of the $T$ axons came from the nasal side of the orbit. And so it was, as shown electrophysiologically and anatomically. ${ }^{7}$ The second approach was to label, with a marker suitable for tracing axons, all or most of the cells in a half of an otherwise normal eye anlage, and then to examine their destination as soon as they arrived in the tectum. It proved to be the 'appropriate' one; i.e. the dorsal tectum for ventral retinal axons. ${ }^{8}$ Finally, the terminal arbors of individual axons were visualized in the tectum with a marker applied to the retina and transported in an anterograde direction to the tectum. They were large, nearly as large as the entire tectum, but biased toward the appropriate quadrant. ${ }^{9}$ Thus it appears that the initial projection is ordered, at least to the accuracy of the retinal quadrant. In a relative sense, the localization of the arbors becomes much more precise with time, as the arbor in the adult occupies only a small fraction of the tectal surface. But in an absolute sense the tectal arbor in the adult is as big, or bigger, than it was early on; the increased precision of localization is a consequence of the increased size of the tectum. ${ }^{9}$

The subsequent stages of development were also first investigated in Xenopus. Straznicky and Gaze showed that cellular proliferation continued in both the retina ${ }^{10}$ and the tectum ${ }^{11}$ of larval Xenopus. The retina grows by adding new annuli, but the tectum adds cells in a topologically dissimilar zone, more like a linear front sweeping caudally and medially. This topologic dissimilarity created a problem in development, since the retinotectal projection was roughly conformal in all larval stages; that is, the center of the retina 


\section{REVIEW ARTICLES}

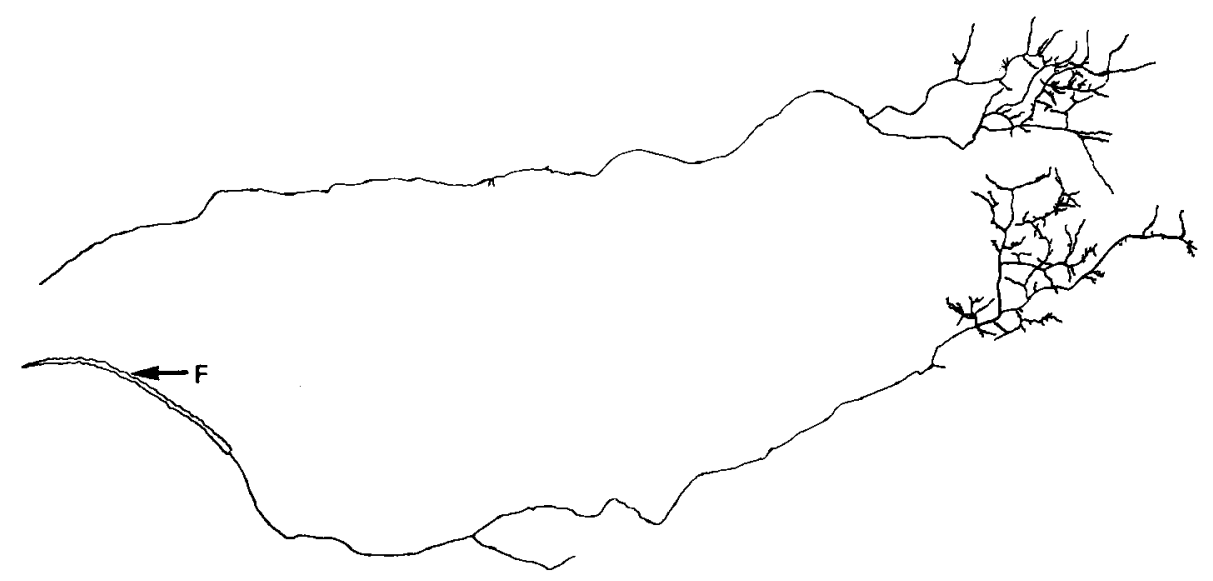

$100 \mu \mathrm{m}$

Fig. 1. Camera lucida drawing of two HRP-labeled retinal axons in the tectum, following application of HRP to the optic nerve. Rostral is to the left, dorsal up, caudal right, ventral down. The lower axon entered in the fascicle $(F)$, then descended into the synaptic layer where it ran caudally to its current termination site. The upper axon followed a similar course but its fascicle is not shown. (From reference 15.)

always projected to the center of the tectum. The central retinal cells were always the same, given the annular growth, but the tectal center was occupied by different cells at all stages because the tectum enlarged caudally. Thus the central retinal cells must have projected to variable partners. A similar argument was developed for other retinal locations, and the 'hypothesis of sliding connections' was born, according to which retinal axons made and broke many synaptic contacts over the course of their development. ${ }^{12}$

This idea has been substantiated in amphibians in two independent experiments, ${ }^{13,14}$ and in fish as well. ${ }^{15,16}$ In fish, moreover, we now know the detailed sequence of events followed by the axons in the development of the retinotectal projection, and those events are described in the remainder of this article. They imply a new set of 'rules' of retinotectal mapmaking, but unlike the rules laid down in the second paragraph of this paper, the new ones specify axonal outgrowth.

\section{The New Rules of Axonal Outgrowth}

Electron microscopic examination of the outgrowing axo.ss in fish has revealed that each generation, whether in the first year or the fifth, behaves similarly. The new ganglion cells, produced at the very edge of the retina, send their axons toward the optic disk in the most superficial sublayer of the fiber layer. They lie directly beneath the inner limiting membrane, in contact superficially with the basal lamina, and deeply with the axons that preceded them from more central retina. Thus there is an age-related stratification of optic axons, youngest most superficial, oldest deepest. As the axons make a right-angle turn to form the optic nerve and exit from the retina, the optic nerve head acquires a retinotopic order; in cross-section, the most central axons are from the most peripheral retina, and vice versa. ${ }^{17}$ This age-related stereotypy is modified and slightly degraded in the passage of the nerve through the orbit, past the optic chiasm and into the tract. ${ }^{18}$ There the axons rearrange and the unitary tract bifurcates to form the dorsal and ventral 'brachia' of the optic tract, the two arms that envelop the tectum from above and below like the blades of a caliper.

At the bifurcation, the new axons always follow the same route: they grow along the outside of the brachium. This conclusion came from two observations. First, electron microscopic examination of the brachium and the tectum showed that the most peripheral axons were always the unmyelinated ones, and they were known from earlier work to be the new ones, from peripheral retina. ${ }^{18}$ Secondly, individual fascicles in the tectum were labeled with horseradish peroxidase (HRP), a marker that is retrogradely transported into the retinal cell bodies of origin. The cells labeled through the peripheral fascicle were always on the retinal margin, and more central fascicles were connected to more central retinal ganglion cells. ${ }^{19}$ In all cases, the zone of labeled cells was a part of an annulus centered on the optic nerve head; therefore generations of ganglion cells had sent their axons in together and they remained together all the way to the tectum. Note that this pathway around the tectal edge is a very efficient one from the perspective of mapmaking; in the topographic projection, the edge of the retina maps to the edge of the tectum. Therefore, by going to the edge of the tectum, the new axons are adjacent to the zone in which they will terminate. At this location, all they need do is exit the brachium at the correct site and they will be in the retinotopically correct tectal site.

This two-dimensional mapping problem is usually considered in terms of the polar coordinates, $r$ and $\theta$, referred to the optic nerve head as the geometric origin. In geometric terms, it is as if the axons solved the problem of termination one dimension at a time. The first choice, to go to the edge, gets them on the right circle, thus they have acquired the appropriate value of $r$. How do they manage to get to the correct value of $\theta$ ? It seemed possible, given the evidence from regeneration, that the choice of $\theta$ might be a disorganized process. That is, regenerating axons follow circuitous pathways to their ultimate (correct) termination sites, apparently making many mistakes along the way. ${ }^{20}$ The prediction from regeneration proved to be inaccurate for normal development. This was shown by labeling (with HRP) the most peripheral fascicle, in a number of fish, at different sites and then examining the retina for labeled somata. All arcs extended from the nasal boundary of the dorsal and ventral hemiretinas around toward the temporal boundary, but depending on where the label was applied, the temporal extent varied. Rostral application of HRP labeled cell bodies well into temporal retina, but the more caudally the HRP was applied, the shorter was the arc of labeled cells. The application of HRP to the fascicle labels all cell bodies with axons still in the fascicle. Since we know that all cells in the half annulus originally had their axons in the fascicle, then the unlabeled region of the partial annulus must include those ganglion cells whose axons had been in the fascicle but exited from it rostral to the application site. Thus the axons exit sequentially, temporal ones first, nasal last. This orderly entry into the tectum assures that the axons arrive at the correct tectal value of $\theta$. Recently obtained results show that the order of exit correlates with the stratigraphic order in the brachium. ${ }^{21}$ 
REVIEW ARTICLES

These two rules of axonal outgrowth, the first of which establishes $r$ and the second $\theta$, create a retinotopic map on the rim of the tectum. But the same topological disparity of growth exists in fish as in amphibians. The fish retina adds annuli, and the tectum adds crescents, with a lot of cells added on the caudal rim, fewer dorsally and ventrally, and none rostrally. Yet the retinotectal projection is similar in both small and large fish (e.g. central retina to central tectum, temporal retina to rostral tectum), so terminals are presumed to shift locations with growth, as in amphibians. This shift can be visualized better in fish than in amphibians because of our detailed knowledge of axonal pathways. If the axons entered in a fascicle around the outer boundary of the tectum, formed their initial terminals just inside this boundary, and later shifted, then the axon would be expected later on to have three fairly distinct regions: (1) in the fascicle, (2) in the synaptic layer passing through various sites where it once terminated, and (3) in the current terminal arbor. Such axons were seen, labeled with HRP, in tectal whole mounts (Fig. 1). Note that much of these axons' extent is in regions of the tectum that did not exist when the axons first grew in, strong evidence that the terminal must have moved. ${ }^{15,16}$

With our understanding of tectal growth and the course of retinotectal axons, we could also predict which retinal axons had passed through any tectal address. These predictions could be tested by retrogradely labeling retinal ganglion cells from individual tectal sites. The predicted results, complex and otherwise counter-intuitive patterns of labeled somata in the retina, were observed (Fig. 2). ${ }^{15}$

Thus the rules of retinotectal mapmaking that are followed by the vast majority of retinal axons, those arriving after the initial contacts are made, are the following. (1) Grow (in association with other new axons) in the space between other recently produced axons on one side and the pia on the other; (2) having chosen the correct brachium, grow along the edge of the tectum; (3) exit the brachium into the tectum in the order dictated by retinal origins, temporal axons first, then progressively more nasal; (4) change terminations in the tectum, maintaining the 'normal' rules of topography.

This last 'rule' is no better understood than the others, but we suppose that it involves inter-axonal competition. If all axons had an intrinsic tendency to

(a)
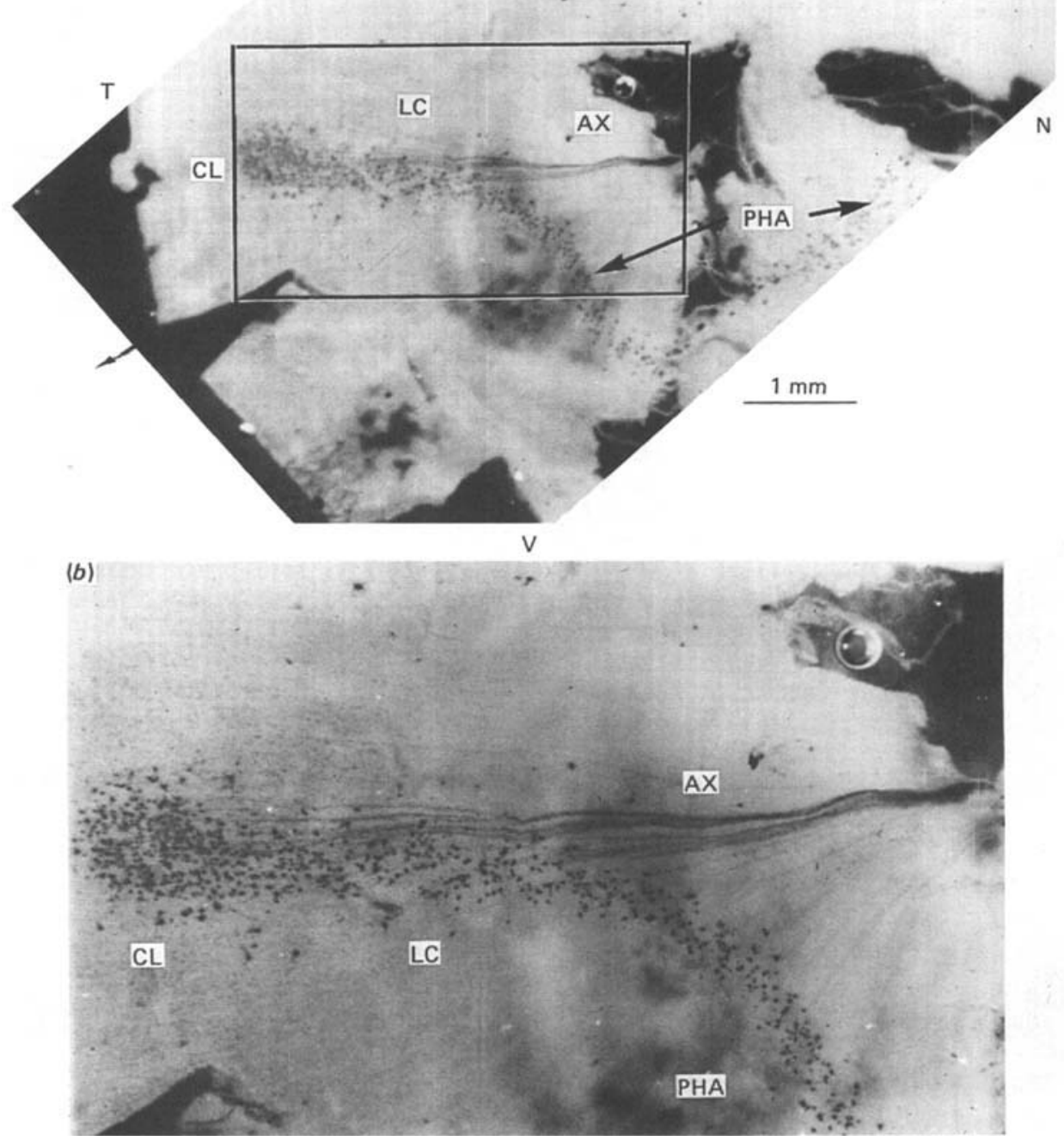

Fig. 2. Photomicrographs of a retinal whole-mount showing HRP-labeled ganglion cells and axons, following punctate application of HRP to the tectum. (a) The original optic nerve head lay at the right edge of the bundle of axons $(A X)$. The labeled ganglion cells are found in three zones, a partial half annulus $(P H A)$ centered on the disk, a linear connector $(L C)$, and a cluster $(C L)$ at the end. The cells in the PHA were labeled through fascicular axons, those in LC through the axons of passage in the synaptic layer, and those in $C L$ through their terminal arbors. (Compare Fig. 1 ; from reference 15. )

enlarge their arbors, and if arbors competed with one another for something in limited quantity, and if the larger arbors competed less well than the smaller ones, then terminals would slide as we have found. There is much to suggest a role for competition, perhaps involving electrical activity, in the spacing of arbors, but that is a large story in itself. ${ }^{22}$

This set of rules is descriptive - no underlying mechanism is proposed. It seems that the phenomena are well enough described now to make one more optimistic that the mechanisms may soon be revealed.

My research is funded by the National Eye Institute of the NIH, through grant EY-00168, for which I am grateful.

\section{REFERENCES}

1 Beazley, L. D. (1984). Formation of specific synaptic connections in the visual system of lower vertebrates. In Current Topics in Research on Synapses, vol. I (ed. D. G. Jones), pp. 53-118. Alan R. Liss, New York.

2 Sperry, R. W. (1963). Chemoaffinity in the orderly growth of nerve fiber patterns and connections. Proc. Natl. Acad. Sci. USA 50, 703-710.

3 Easter, S. S., Purves, D., Rakic, P. \& SpITZER, N. C. (1985). The changing view of neural specificity. Science 230, 507-511.

4 Trisler, G. D., Schneider, M. D. \& NirenberG, M. (1981). A topographic gradient of molecules in retina can be used to identify neuron position. Proc. Natl. Acad. Sci. USA 78, 2145-2149.

5 BonHOEFFER, F. \& HuF, J. (1982). In vitro experiments on axon guidance demonstrating an anterior-posterior gradient on the tectum. EMBO J. 1, 427-431. 


\title{
REVIEW ARTICLES
}

6 Edelman, G. M. (1983). Cell adhesion molecules. Science 219, 450-457.

7 Straznicky, C., Gaze, R. M.\& Keating, M. J. (1981). The development of the retinotectal projections from compound eyes in Xenopus. J. Embryol. Exp. Morphol. 62, 13-35.

8 Holt, C. E. \& Harris, W. A. (1983) Order in the initial retinotectal map in Xenopus: a new technique for labelling growing nerve fibres. Nature 301, 150-152. 9 Sakaguchi, D. S. \& Murphey, R. K. (1985). Map formation in the developing Xenopus retinotectal system: an examination of ganglion cell terminal arborizations. J. Neurosci. 5, 3228-3245.

10 Straznicky, C. \& Gaze, R. M. (1971). The growth of the retina in Xenopus laevis: an autoradiographic study. J. Embryol. Exp. Morphol. 26, 67-79.

11 StrazniCKy, C. \& Gaze, R. M. (1972). The development of the optic tectum in Xenopus laevis: an autoradiographic study. J. Embryol. Exp. Morphol. 28, 87-115.

12 Gaze, R. M., Keating, M. J. \& ChUnG, S. H. (1974). The evolution of the retinotectal map during development in Xenopus. Proc. Roy. Soc. London, B 185, 301-330.
13 Constantine-Paton, M., Pitts, E. C. \& REH, T. A. (1983). The relationship between retinal axon ingrowth, terminal morphology, and terminal patterning in the optic tectum of the frog. J. Comp. Neurol. 218, 297-313.

14 Fraser, S. E. (1983). Fiber optic mapping of the Xenopus visual system: shift in the retinotectal projection during development. Dev. Biol. 95, 505-511.

15 Easter, S. S. \& Stuermer, C. A. O. (1984). An evaluation of the hypothesis of shifting terminals in goldfish optic tectum. J. Neurosci. 4, 1052-1063.

16 Rusofr, A. C. (1984). Paths of axons in the visual system of perciform fish and implications of these paths for rules governing axonal growth. J. Neurosci. 4, 1414-1428.

17 Easter, S. S., Bratton, B. \& Scherer, S. S. (1984). Growth-related order in the retinal fiber layer in goldfish. $J$. Neurosci. 4, 2173-2190.

18 Easter, S. S., Rusoff, A. C. \& Kish, P. E. (1981). The growth and organization of the optic nerve and tract in juvenile and adult goldfish. J. Neurosci. 1, 793-811.

19 Stuermer, C. A. O. \& Easter, S. S.
(1984). Rules of order in the retinotectal fascicles of goldfish. $J$. Neurosci. 4, 1045-1051.

20 HORDER, T. J. (1974). Changes of fiber pathways in the goldfish optic tract following regeneration. Brain Res. 72, 41-52.

21 Bernhardt, R. \& EASTER, S. S. (1986). The map of retinal position onto the cross section of the optic pathway of goldfish. J. Comp. Neurol. (in press).

22 SCHMIDT, J. T. (1985). Factors involved in retinotopic map formation: complementary roles for membrane recognition and activity-dependent stabilization. In Molecular Bases of Neural Development (ed. G. M. Edelman, W. E. Gall \& W. M. Cowan), pp. 453-480. Wiley, New York.

STEPHEN S. EASTER, JR. is in the Division of Biological Sciences, 2109 Natural Science Building, University of Michigan, Ann Arbor, MI 48109, USA.

\section{Interstrand Duplexes in Nuclear RNA}

\author{
A. Oscar Pogo and Kenton S. Miller
}

\section{Summary}

Nuclear intermolecular duplexes appear to be a general feature of nucleated cells. Most of these duplexes are formed between large RNA as well as between large and small RNA molecules. A significant portion of the large molecules belong to a special class of RNA that is restricted to the nucleus and, therefore, not designated for export. These molecules are assembled with proteins and form a structure of a higher order. The possibility that these molecules and a set of small nuclear RNAs are components of a complex machine, 'the transportosome', which functions in nucleocytoplasmic traffic, is discussed.

\section{Introduction}

In recent years RNA has changed its image and become the most versatile cellular entity. Its reputation has evolved from that of a passive communicator of information, a mere 'adapter' in the decoding process, a humble half of the ribosomal mass, to serve as an active instrument in biochemical processes. In particular, RNA can be the executant of phosphodiester cleavage and ligation in the absence of protein. The folded RNA molecule is capable of self-splicing by this cleavage-ligation process, ${ }^{1}$ a reaction that had been attributed to enzymes exclusively. The RNA moiety of ribonuclease $P$, the enzyme responsible for cleaving transfer RNA (tRNA) precursors to generate the mature $5^{\prime}$ end of the tRNA, is another example of an RNA molecule that meets the criteria of an enzyme. ${ }^{2}$ The intervening sequence of the Tetrahymena ribosomal RNA (rRNA) precursor is also a true enzyme; 3 this RNA 'enzyme' catalyzes in vitro, in the absence of proteins, the cleavage and rejoining of oligonucleotide substrates in a sequence-dependent manner. Thus this RNA molecule can act as an RNA polymerase, differing from the protein enzyme in that it uses an internal rather than an external template. Therefore the RNA molecule may be the essential principle of life; its creation could have been the start of it all.

\section{RNA as Regulators of Gene Expression}

Could it also be that RNA is a regulator of gene expression? RNA as the repressor in bacteria was postulated by Jacob and Monod more than two decades ago. ${ }^{4}$ It is interesting that the prediction of the chemical nature of the repressor was based upon the negative evidence available then that it was not a protein. We now know, however, that repressors in bacteria are proteins, but, the discovery of complementary repetitive sequences on pre-messenger RNA molecules has revived the idea that RNA molecules somehow can act as gene regulators. Davidson and Britten pioneered the concept that the formation of RNA-RNA duplexes via complementary repetitive sequences could be a mechanism for messenger RNA (mRNA) processing. ${ }^{5}$ However, the detailed structural analysis of intron-exon (intron or noncoding sequences; exon or coding sequences) splicing junctions and their flanking sequences does not reveal the presence of such comple- 